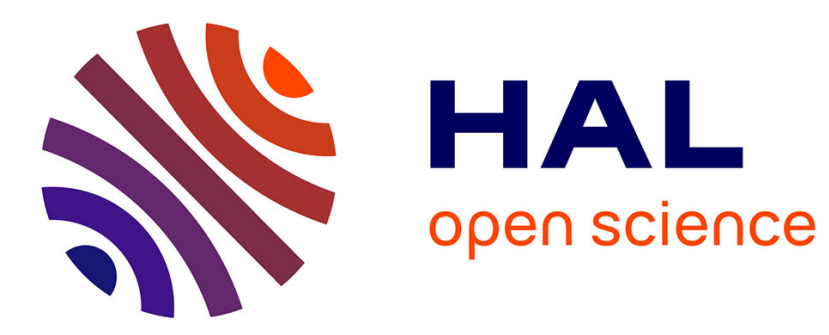

\title{
Acoustic characterization of a nonlinear vibroacoustic absorber at low frequencies and high sound levels
}

Alice Chauvin, Mélodie Monteil, Sergio Bellizzi, Renaud Côte, Philippe

Herzog, M Pachebat

\section{- To cite this version:}

Alice Chauvin, Mélodie Monteil, Sergio Bellizzi, Renaud Côte, Philippe Herzog, et al.. Acoustic characterization of a nonlinear vibroacoustic absorber at low frequencies and high sound levels. Journal of Sound and Vibration, 2018, 416, pp.244-257. 10.1016/j.jsv.2017.11.031 . hal-01636357

\section{HAL Id: hal-01636357 \\ https://hal.science/hal-01636357}

Submitted on 2 May 2018

HAL is a multi-disciplinary open access archive for the deposit and dissemination of scientific research documents, whether they are published or not. The documents may come from teaching and research institutions in France or abroad, or from public or private research centers.
L'archive ouverte pluridisciplinaire HAL, est destinée au dépôt et à la diffusion de documents scientifiques de niveau recherche, publiés ou non, émanant des établissements d'enseignement et de recherche français ou étrangers, des laboratoires publics ou privés. 


\title{
Acoustic characterization of a nonlinear vibroacoustic absorber at low frequencies and high sound levels
}

\author{
A. Chauvin, M. Monteil, S. Bellizzi, R. Côte*, Ph. Herzog, M. Pachebat \\ Aix Marseille Univ, CNRS, Centrale Marseille, LMA, Marseille, FRANCE
}

\begin{abstract}
A nonlinear vibroacoustic absorber (Nonlinear Energy Sink: NES), involving a clamped thin membrane made in Latex, is assessed in the acoustic domain. This NES is here considered as an one-port acoustic system, analyzed at low frequencies and for increasing excitation levels. This dynamic and frequency range requires a suitable experimental technique, which is presented first. It involves a specific impedance tube able to deal with samples of sufficient size, and reaching high sound levels with a guaranteed linear response thank's to a specific acoustic source. The identification method presented here requires a single pressure measurement, and is calibrated from a set of known acoustic loads. The NES reflection coefficient is then estimated at increasing source levels, showing its strong level dependency. This is presented as a mean to understand energy dissipation. The results of the experimental tests are first compared to a nonlinear viscoelastic model of the membrane absorber. In a second step, a family of Helmholtz resonator is identified from the measurements, allowing a parametric description of the NES behavior over a wide range of levels.
\end{abstract}

Keywords: Passive Absorber, Acoustic Impedance Measurement, Impedance Tube, High Level, Low Frequency

\section{Introduction}

Nonlinear vibroacoustic absorbers are passive devices dedicated to noise reduction at low frequencies. Such devices consist in a thin structure submitted to large deformations, which exhibits non linear resonances used to absorb sound energy. Such a structure is a thin viscoelastic membrane in 1, whereas in 2] the diaphram of a loudspeaker without motor assembly is used. This kind of device takes advantage of a phenomenon called "Targeted Energy Transfer" or "Energy Pumping", due to the coupling of the nonlinear resonance of the absorber with the acoustic field that has to be reduced. It is described in detail in 3 in terms of resonance capture and nonlinear modes showing an irreversible energy transfer from the acoustic medium toward the absorber, the energy being thus dissipated within the absorber. In the literature, such a device is often designated as

\footnotetext{
*Corresponding Author

Email address: cote@lma.cnrs-mrs.fr (R. Côte)

Preprint submitted to Journal of Sound and Vibration
}

October 4, 2017 
a "Nonlinear Energy Sink" (NES). Nonlinear vibroacoustic absorbers have shown their ability to reduce sound especially at low frequencies range and high enough sound field levels. They may thus overcome some limitations of classical devices such as porous materials, Helmholtz resonators, perforated plates [1, 2, 4, 5, 6].

In these early papers, the nonlinear behavior of the vibroacoustic absorbers was modelled, using nonlinear plate equations of the Von-Kármán type for the thin structure, coupled with the acoustic medium through an impedance boundary condition. In addition to these models, experiments were based on vibration measurements focused on the mechanical NES behavior. Conversely, the present paper focuses on the NES considered as an acoustic device with desirable absorbing properties. Dealing with low frequencies, it uses an approach common to the characterization of other kinds of absorbers, i.e. a cylindrical tube connecting a sound source to the NES. At the considered frequencies, plane waves may safely be assumed into the tube, so the NES may be considered as a single port acoustic device.

A review about linear impedance measurements can be found in 7 . The most employed method is the Two Microphone Method (TMM). It was initially developed by Seybert and Ross 8 and expanded by Chung and Blaser using transfer functions [9, 10. Abom and Boden [11] later reviewed different implementations of this method, the most usual being standardized as ISO 10354-2. Basically, it involves two microphones placed between the sample and the source, their relative calibration requiring them to be switched. The microphones spacing must be adapted to the targeted frequency band, which becomes problematic when dealing with wide frequency ranges. An improvement of the calibration method, using three loads, was later proposed by Gibiat and Laloë [12]. Recently, Boonen et al. 13. proposed a method to deal with high impedance magnitudes over a wide frequency range : the calibration is then again performed by measuring several different acoustic loads instead of switching the microphones.

In our study, the absorber is essentially nonlinear and its absorbing properties have to be dug out at low frequencies, under controlled high excitation levels. No standard equipment is available to deal with this need, so we built an impedance tube (thereafter named "Short Kundt's Tube", i.e. SKT) able to reach very high levels at low frequencies (typically several hundreds of Pascals between 10 and $200 \mathrm{~Hz}$ ). The measurement of the acoustic characteristics of the absorber device are obtained through an original method inspired from the indirect source characterization methods proposed in [14, 15, 16. A single microphone is needed, as the source is first characterized using different known acoustic loads. An "equivalent impedance" and the apparent reflection coefficient of the NES are then deduced from this single pressure measurement.

The SKT is named "short" because it is shorter than should be a Kundt's tube according to ISO 10354-2. A single microphone method can use a tube as short as possible with respect to the plane waves approximation. Such a short device is easy to move and occupies a small area. With this setup we expect to reduce errors due to thermal gradient and instabilities along the tube, and we expect to reject the source resonances above the measurement range, as shown in this article.

The structure of this paper is as follows. Section 2 presents the experimental set-up and the identification method allowing to estimate the reflection coefficient of a device under study (DUT). Section 3 applies this method to the estimation of the reflection coefficient of a sample nonlinear absorber, and its measurements are then compared to an analytic model previously published (Section 3.3 ) and to a set of equivalent resonators 
(Section 3.4).

In this paper, all quantities, unless otherwise stated, will be considered in the frequency domain, assuming a $e^{+\mathrm{j} \omega t}$ temporal dependence.

\section{Measurement method}

(a)

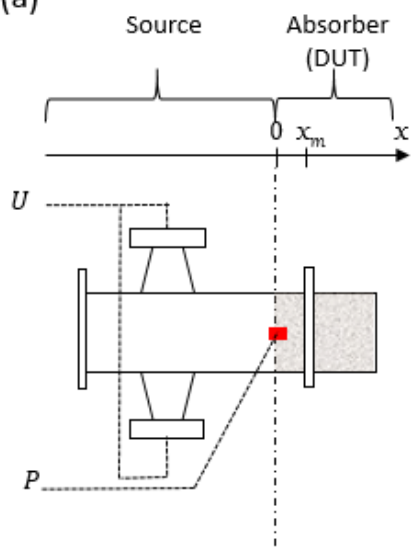

(b)

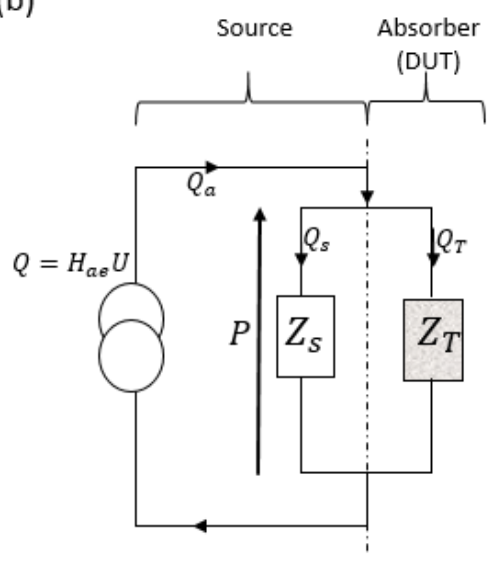

Figure 1: Scheme of (a) the experimental set-up and (b) the equivalent electroacoustic circuit of the one-microphone identification method.

\subsection{Basic principle}

Fig. 1(a) presents the acoustical scheme of the set-up. It is made of a sound source (controlled by a voltage $U$ ) and a sample (or DUT), connected through a tubular section of minimal length (hence the name "Short" Kundt's Tube). The source must be linear over the full dynamic range, and therefore features several driver units (only two are shown in Fig. 1(a)). The left hand side of the tube is closed next to the source, and the DUT is mounted on the right hand side. A high pressure microphone is positioned on the axis of the tube, in the measurement plane separating the source from the DUT which is taken as the origin for the $x$-axis.

The DUT is characterized by an equivalent impedance $Z_{\mathrm{T}}$, which is defined as the ratio of the fundamental components of the spectra of the pressure and the volume velocity, both considered over the tube section in the measurement plane. Plane waves are assumed along the cylindrical part of the system, at sufficient distances from the source and the DUT: the measurement plane is distant at least of the magnitude of one tube diameter from the loudspeaker connectors, and one membrane diameter from the sample membrane. Tests were made with a $0.8 \mathrm{~m}$ long extension of the tube between the DUT and the microphone, and no significant difference was noticed between the results. The local pressure $P$ measured by the microphone is thus considered as a good estimate of the pressure averaged over the measurement section. The local volume velocity is not measured, but is indirectly obtained through the setup calibration. When the DUT 
is nonlinear, $Z_{\mathrm{T}}$ corresponds to a linear approximation of the relationship between the pressure and volume velocity. In this case, $Z_{\mathrm{T}}$ is an indicator depending on the DUT, obviously, and depending also of the conditions of the experiment (i.e. $U$ and the source frequency content).

The source is considered as linear and time-invariant, so it can be represented using the Norton equivalence by the electro-acoustic scheme shown in Fig. 1(b). It is characterized by its impedance $Z_{\mathrm{S}}$ and its volume velocity $Q_{\mathrm{a}}$. Equivalently, it may be characterized by $Z_{\mathrm{s}}$ and the transfer function $H_{a e}$ between the acoustic volume velocity $Q_{\mathrm{a}}$ and the control voltage $U$, defined as $Q_{\mathrm{a}}=H_{\mathrm{ae}} U$. The equivalent impedance $Z$ loading the volume velocity source is therefore :

$$
Z=\frac{Z_{\mathrm{T}} Z_{\mathrm{s}}}{Z_{\mathrm{T}}+Z_{\mathrm{s}}}
$$

Considering the transfer function $H_{m}$ between the control voltage $U$ of the source and the acoustic pressure $P$ at the microphone, Eq. (1) reduces to :

$$
H_{\mathrm{m}}=\left(\frac{1}{Z_{\mathrm{s}}}+\frac{1}{Z_{\mathrm{T}}}\right)^{-1} H_{\mathrm{ae}}
$$

and the impedance $Z_{\mathrm{T}}$ and the reflection coefficient $R_{\mathrm{T}}$ of the DUT can be expressed as :

$$
Z_{\mathrm{T}}=\frac{H_{\mathrm{m}} Z_{\mathrm{s}}}{H_{\mathrm{ae}} Z_{\mathrm{s}}-H_{\mathrm{m}}} \text { and } R_{\mathrm{T}}=\frac{H_{\mathrm{m}} Z_{\mathrm{s}}-Z_{\mathrm{c}}\left(Z_{\mathrm{s}} H_{\mathrm{ae}}-H_{\mathrm{m}}\right)}{H_{\mathrm{m}} Z_{\mathrm{s}}+Z_{\mathrm{c}}\left(Z_{\mathrm{s}} H_{\mathrm{ae}}-H_{\mathrm{m}}\right)}
$$

where $Z_{\mathrm{c}}=\rho c / S$ is the specific acoustic impedance of air, $\rho$ is the density of air and $c$ the sound wave velocity.

Thus, the determination of the impedance $Z_{\mathrm{T}}$ (and the reflection coefficient $R_{\mathrm{T}}$ ) of the DUT only requires transfer function $H_{m}$ measurement and the prior knowledge of the source characteristics $H_{a e}$ and $Z_{\mathrm{s}}$. This will be discussed in the following two sections.

Note that $Z_{\mathrm{T}}$ and $R_{\mathrm{T}}$ are defined at the microphone axial position but they can be deduced at the location of the load thanks to the impedance translation theorem between two axial positions $x_{1}$ and $x_{2}$ in the tube, recalled hereafter :

$$
\left(\begin{array}{l}
P\left(x_{2}\right) \\
Q\left(x_{2}\right)
\end{array}\right)=\left(\begin{array}{cc}
\cos \left(k\left(x_{1}-x_{2}\right)\right) & Z_{c} \mathrm{j} \sin \left(k\left(x_{1}-x_{2}\right)\right) \\
Z_{c}^{-1} \mathrm{j} \sin \left(k\left(x_{1}-x_{2}\right)\right) & \cos \left(k\left(x_{1}-x_{2}\right)\right)
\end{array}\right)\left(\begin{array}{l}
P\left(x_{1}\right) \\
Q\left(x_{1}\right)
\end{array}\right)
$$

where $k=\omega / c$ is the wavenumber.

Combining the source characteristics $H_{\mathrm{ae}}$ and $Z_{\mathrm{s}}$ and the load impedance $Z_{\mathrm{T}}$, it is therefore possible to estimate the transfer function between control voltage $U$ and the acoustic pressure at a new axial position $x_{\mathrm{n}}$ as :

$$
H_{\mathrm{m}}^{\mathrm{n}}=\frac{Z_{\mathrm{s}}^{\mathrm{n}} Z_{\mathrm{T}}^{\mathrm{n}}}{Z_{\mathrm{s}}^{\mathrm{n}}+Z_{\mathrm{T}}^{\mathrm{n}}} H_{\mathrm{ae}}^{\mathrm{n}}
$$

where

$$
Z_{\mathrm{T}}^{\mathrm{n}}=\frac{\cos \left(-k x_{\mathrm{n}}\right)+\mathrm{j} \sin \left(-k x_{\mathrm{n}}\right) Z_{\mathrm{c}} / Z_{\mathrm{T}}}{\mathrm{j} \sin \left(-k x_{\mathrm{n}}\right) / Z_{\mathrm{c}}+\cos \left(-k x_{\mathrm{n}}\right) / Z_{\mathrm{T}}}, Z_{\mathrm{s}}^{\mathrm{n}}=\frac{\cos \left(k x_{\mathrm{n}}\right) Z_{\mathrm{s}}+\mathrm{j} Z_{\mathrm{c}} \sin \left(k x_{\mathrm{n}}\right)}{\cos \left(k x_{\mathrm{n}}\right)+\mathrm{j} \sin \left(k x_{\mathrm{n}}\right) Z_{\mathrm{s}} / Z_{\mathrm{c}}},
$$




$$
H_{\mathrm{ae}}^{\mathrm{n}}=\left(\cos \left(k x_{\mathrm{n}}\right)+j Z_{\mathrm{c}} \sin \left(k x_{\mathrm{n}}\right) Z_{\mathrm{s}}^{\mathrm{n}^{-1}}\right) H_{\mathrm{ae}} .
$$

Transformation (5) will be used for the comparison with mechanical models of the NES in Section 3 .

\subsection{Setup calibration}

The setup calibration consists in determining the quantities $H_{\text {ae }}$ and $Z_{\mathrm{s}}$, from measurements of known acoustic loads. In such a case, Eq. (2) becomes :

$$
H_{\mathrm{m}}=\frac{Z_{\mathrm{s}} Z_{\mathrm{T}}}{Z_{\mathrm{s}}+Z_{\mathrm{T}}} H_{\mathrm{ae}}
$$

The measurements $H_{\mathrm{m}_{1}}$ and $H_{\mathrm{m}_{2}}$ obtained respectively from two different (but known) loads $Z_{\mathrm{T}_{1}}$ and $Z_{\mathrm{T}_{2}}$ are thus theoretically sufficient to determine the source parameters $H_{\mathrm{ae}}$ and $Z_{\mathrm{s}}$ as :

$$
H_{\mathrm{ae}}=\frac{H_{\mathrm{m}_{1}} H_{\mathrm{m}_{2}}\left(Z_{\mathrm{T}_{1}}-Z_{\mathrm{T}_{2}}\right)}{Z_{\mathrm{T}_{1}} Z_{\mathrm{T}_{2}}\left(H_{\mathrm{m}_{1}}-H_{\mathrm{m}_{2}}\right)} \text { and } Z_{\mathrm{s}}=\frac{Z_{\mathrm{T}_{1}} Z_{\mathrm{T}_{2}}\left(H_{\mathrm{m}_{1}}-H_{\mathrm{m}_{2}}\right)}{H_{\mathrm{m}_{2}} Z_{\mathrm{T}_{1}}-H_{\mathrm{m}_{1}} Z_{\mathrm{T}_{2}}} .
$$

Since the transfer functions $H_{\mathrm{m}_{1}}$ and $H_{\mathrm{m}_{2}}$ are obtained using the same microphone, $Z_{\mathrm{s}}$ (given by Eq. (9)) does not depend on the microphone calibration. The same property applies to the determination of $Z_{\mathrm{T}}$ (given by Eq. (3)). Hence with this approach the microphone calibration is not needed.

A simple sensitivity study of Eqs. (9) brings some observations. For frequencies where $H_{\mathrm{ae}}=0, Z_{\mathrm{s}}=0$ or $H_{\mathrm{m}}=0$, the load impedance cannot be estimated. Moreover, as pointed out by Bodén et al. [17, if $Z_{\mathrm{T}_{1}}$ and $Z_{\mathrm{T}_{2}}$ are close to each other, the error on $Z_{\mathrm{s}}$ prevents a reliable calibration. Conversely, when $Z_{\mathrm{s}}$ is of the same order of magnitude as the load impedance $Z_{\mathrm{T}}$, the error in the estimation of $H_{\mathrm{ae}}$ is smaller.

In order to avoid errors and improve the results, an over-determination of the calibration data is therefore desirable. It can be performed by combining more than two known loads, a technique which is briefly summarized in Appendix A.

\subsection{Setup consistency}

A very basic assumption behind the proposed method is that the source is assumed linear and time-invariant. Its specific electroacoustic design ensures that it may deliver a high volume velocity, required at low frequencies, while keeping a sufficient margin compared to the maximal displacement specified by the loudspeakers manufacturer. Care has also been taken in order to minimize parasitic nonlinear behaviors related to high local acoustic over-pressures, rapid air flows around edges, leakages, etc. Last, the electric powers required during the NES measurements are significantly smaller than the thermal limits specified for the voice-coil heating of loudspeakers, thus reducing the risk of thermal compression during the measurements. A brief description of the source design is given in Appendix B.

Beyond these basic design steps, the source linearity has been checked thoroughly by increasing the RMS level when measuring linear acoustic loads. No significant discrepancy has been found between measurements at different levels, strenghtening the hypothesis of a linear setup. A set of calibration loads has been designed, and used within 
a minimization process in order to determine a suitable number of loads for calibration. This led to using six reference loads (see Appendix C) spanning a wide impedance range. The full description of these technical aspects is beyond the scope of the present paper, and only cited here to back up the measurement results presented in this paper.

It should be noted that the above-mentionned calibration process is started before every measurements series, as small discrepancies have been detected between calibrations performed at several days intervals. The loudspeaker behavior impacts the calibration data. Such high-power devices do not have the same stability over time than measurement sensors. Frequent calibrations are therefore mandatory to maintain a sufficient measurement accuracy. This time consuming and as such a limitation of our approach.

\section{Nonlinear vibroacoustic absorber behavior}

\subsection{Description of the vibroacoustic absorber}

The nonlinear vibroacoustic absorber considered in this study (hereafter named NES) has been described in detail by Bellet et al. 1, 4]. It consists in a thin viscoelastic circular membrane (see Fig. 2(a)) clamped by a sliding system, allowing to apply a constant inplane pre-stress to the membrane. When the membrane is subjected to a large acoustic pressure, its flexural displacement can be several orders of magnitude larger than the membrane thickness, resulting in a nonlinear behavior. This NES is mounted at the end of the source as illustrated by Fig. 2(b). The measurements presented below were made using a membrane made of latex, with a diameter equal to $0.06 \mathrm{~m}$ and a thickness equal to $1.810^{-4} \mathrm{~m}$.

(a)

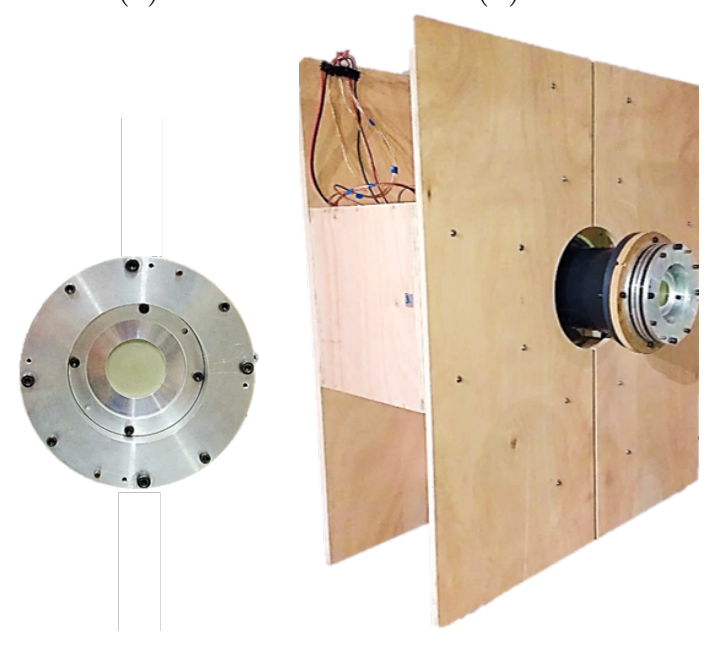

Figure 2: (a) Front view of the NES (sliding system and membrane). (b) NES mounted on the source (with a sight of the sliding system). 


\subsection{Acoustic characterization of the NES}

The acoustic characterization of the NES was carried out using [10,200] $\mathrm{Hz}$ bandlimited white noise with a control voltage $U$. A multi-channel analyzer/recorder (OROS OR38) including a signal generator was used with a sampling frequency of $3200 \mathrm{~Hz}$. The estimations of the transfer function $H_{\mathrm{m}}$ were obtained with a $\Delta f=0.5 \mathrm{~Hz}$ frequency resolution corresponding to 2 sec time length segments and 50 averages. Seven excitation levels were considered starting from $U_{\mathrm{RMS}}=0.01 \mathrm{~V}$ to $11.68 \mathrm{~V}$, resulting in acoustic pressures between $2 \cdot 10^{-2} \mathrm{~Pa}$ and $350 \mathrm{~Pa}$ at the microphone position (see Fig 3). We chose white noise excitation because it allows fast measurements and because it is representative of some NES application environments (e.g. room noise absorption). The NES is nonlinear, some transient state may be several orders of magnitude longer than the period of the excitation and exhibit hysteresis (e.g. SMR 3), so a frequency stepped excitation may take a long time, and a chirped excitation may give results difficult to understand. Note that these measurements on a nonlinear DUT are not a priori representative of other excitation schemes. Nevertheless, our hypothesis is that this excitation and an analysis based on transfer functions should give a relevant characterization of a NES. It would be interesting in a future work to further study the temporal dynamics of the NES response.
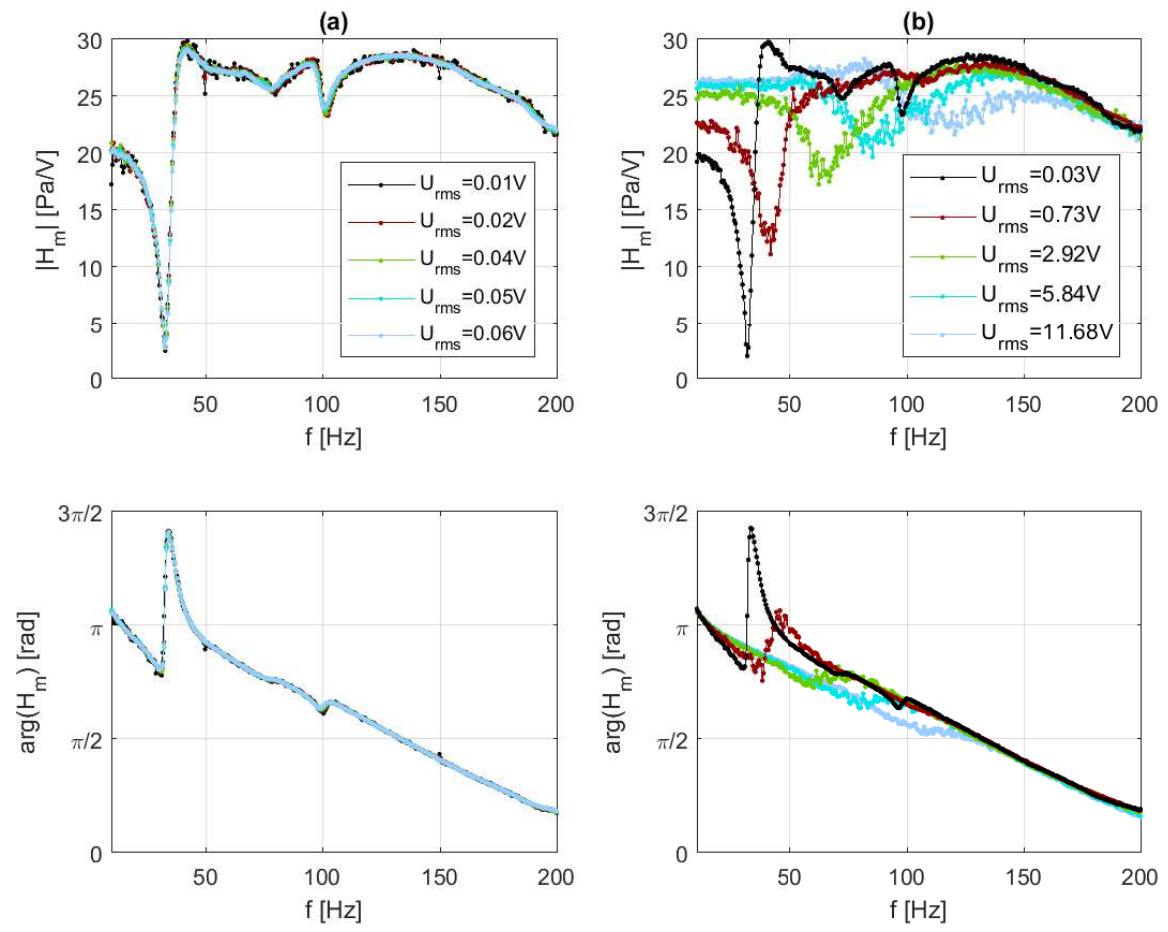

Figure 3: Measured NES transfer function $H_{\mathrm{m}}$ for several excitation levels: modulus(up) and phase (down). (a) Low excitation level (linear behavior) ; (b) High excitation level (nonlinear behavior). 
Figure 3 shows modulus and phases of the transfer function $H_{\mathrm{m}}$ measured at the seven RMS values of the control voltage $U$.

At low excitation levels, $U_{\mathrm{RMS}}=0.01 \mathrm{~V}$ and $0.02 \mathrm{~V}$, the system shows a linear behavior as observed in Fig. 3(a). The transfer function exhibits a minimum near $30 \mathrm{~Hz}$. This minimum of pressure within the tube corresponds to a maximum of the membrane motion characterizing the linear resonance frequency of the NES (at low excitation levels). A nonlinear behavior appears when increasing the excitation level, see Fig. 3(b). The resonance frequency is shifted toward higher frequencies with a broadening resonance dip. This is characteristic of a system hardening with level.

For each excitation level, the acoustic impedance $Z_{\mathrm{T}}$, Eq. (3), and the corresponding reflection coefficient $R_{\mathrm{T}}$, Eq. (3), can be deduced from the measured transfer function $H_{\mathrm{m}}$ and the estimated source characteristics $H_{\mathrm{ae}}$ and $Z_{\mathrm{s}}$ (calibration process). The calibration process was carried out before the measurement series of the NES and using the six reference loads (see Appendix C). The results are reported Fig. 4 showing a smooth and flat behavior of $H_{\mathrm{ae}}$ and $Z_{\mathrm{s}}$ over the considered frequency range. The source is linear without obvious resonances over the frequency range of the study. As a consequence, $H_{\mathrm{ae}}$ and $Z_{\mathrm{s}}$ curves should be continuous and smooth. On the actual measurements, each point of these quantities has been estimated independently of the others, and the smoothness of the actual curves is an indication about the quality of the measurements. The flatness of the curves can be viewed as a property of the source. It means that the response of the system has no sharp differences along the frequency range, that resonances of the source do not screen DUT characteristics, or in other words that the sensitivity to measurement errors is similar along the frequency range.

Figure 5(a) shows modulus and phases of $Z_{\mathrm{T}}$ for the five excitation levels considered in Fig. 3(b). A similar nonlinear behavior as the transfer function $H_{\mathrm{m}}$ is observed for $Z_{\mathrm{T}}$. The reflection coefficient $R_{\mathrm{T}}$ (modulus and phases) is shown by Fig. 5 (b). It allows to assess the amount of energy removed out of the system by the termination, compared to the case of a total energy reflection $\left|R_{\mathrm{T}}\right|^{2}=1$. Here, the energy is extracted by the NES over a frequency range widening with the increase of the excitation level. It is important to note that only a part of extracted energy is dissipated (absorbed) by the NES while another part is radiated by the outward face of the membrane or converted to different frequencies [5].

The maximum of extraction of energy $\left(\left|R_{\mathrm{T}}\right| \approx 0.05\right)$ is observed for $U_{\mathrm{RMS}}=5.84 \mathrm{~V}$ at a frequency $f \simeq 80 \mathrm{~Hz}$. Considering these observations, up to $90 \%$ energy can be extracted by the NES near its resonance frequency. Further increase of $U_{\text {RMS }}$ however tends to slightly reduce the energy extracted by the NES.

\subsection{Comparison with a viscoelastic model}

The measurements resulting from the behavior of the NES are now compared to results from the viscoelastic model proposed by Bellet et al. 11. The nonlinear equation of motion of the membrane (NES) is thus obtained considering the membrane as a thin elastic structure with geometric nonlinearities. The transverse displacement of the membrane is described using a one degree of freedom Rayleigh-Ritz reduction with a single parabolic deflection shape function. The nonlinear equation of motion takes the following form :

$$
m_{\mathrm{me}} \ddot{u}_{\mathrm{me}}+k_{1}\left[\left(\frac{f_{1 \mathrm{me}}}{f_{0 \mathrm{me}}}\right)^{2} u_{\mathrm{me}}+\eta \dot{u}_{\mathrm{me}}\right]+k_{3}\left(u_{\mathrm{me}}^{3}+2 \eta u_{\mathrm{me}}^{2} \dot{u}_{\mathrm{me}}\right)=\frac{S_{\mathrm{me}}}{2} p_{\mathrm{me}}(t)
$$


(a)
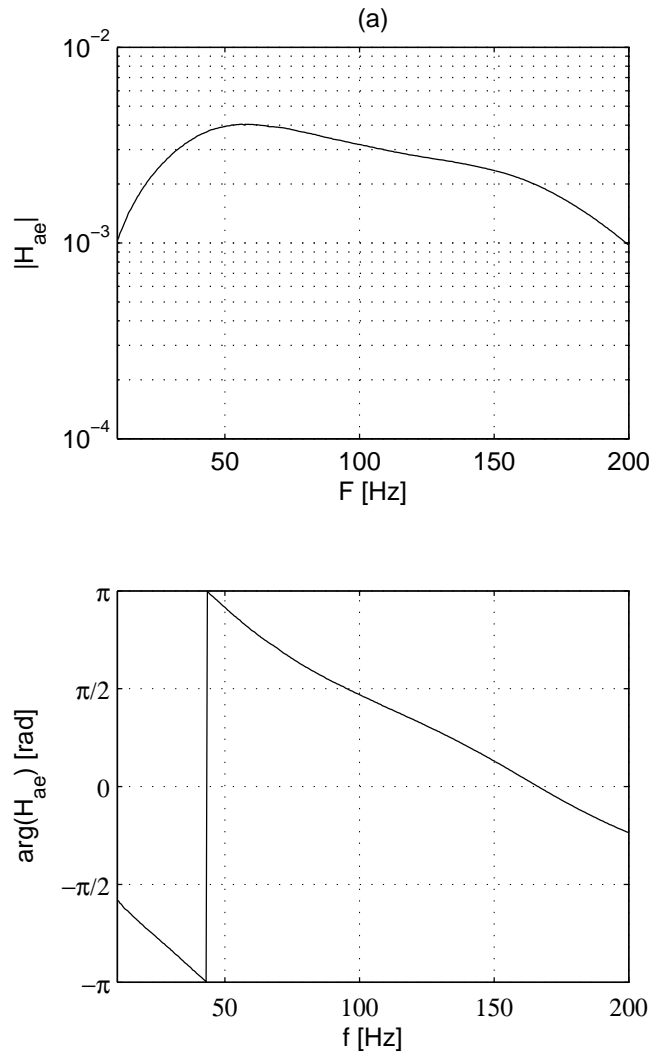

(b)
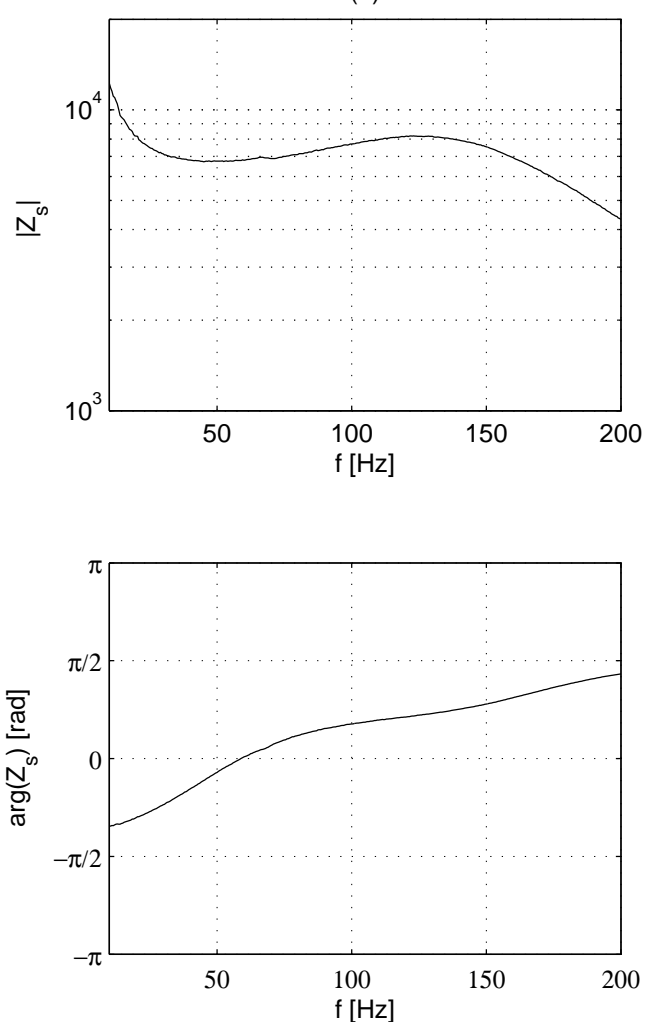

Figure 4: Estimated source parameters $H_{\text {ae }}$ and $Z_{\mathrm{S}}$ using the six reference loads (see Appendix C) Modulus (top) and phase (bottom).

where $u_{\text {me }}$ denotes the (instantaneaous) displacement of the center of the membrane, $p_{\text {me }}$ denotes the resulting acoustic pressure in the vicinity of the membrane inside the tube and the dot represents time-differentiation. The coefficient $1 / 2$ in the right hand side of Eq. (10) results from the parabolic form used in the Rayleigh-Ritz reduction 11. The model parameters are given by :

$$
\begin{gathered}
k_{1}=\frac{2 \pi E h_{\mathrm{me}}^{3}}{3\left(1-\nu^{2}\right) R_{\mathrm{me}}^{2}}, \quad k_{3}=\frac{1}{2} \frac{8 \pi E_{\mathrm{me}} h_{\mathrm{me}}}{3\left(1-\nu^{2}\right) R_{\mathrm{me}}^{2}} \\
m_{\mathrm{me}}=\frac{\rho_{\mathrm{me}} h_{\mathrm{me}} S_{\mathrm{me}}+m_{\mathrm{a}}}{3}, \quad f_{0 \mathrm{me}}=\frac{1}{2 \pi} \sqrt{\frac{1.015^{4} \pi^{4} E h_{\mathrm{me}}^{2}}{12\left(1-\nu^{2}\right) \rho_{\mathrm{me}} R_{\mathrm{me}}^{4}}}
\end{gathered}
$$

where $\rho_{\text {me }}$ is the density, $\nu$ the Poisson's coefficient and $E$ the Young's modulus of the membrane made in latex, $h_{\text {me }}$ is its thickness, $R_{\text {me }}$ its radius and $S_{\text {me }}$ its surface. The coefficients $k_{1}$ and $k_{3}$ respectively stand for the linear and nonlinear stiffnesses. As justified by Bellet et al. [1], a 1/2 coefficient was included in the expression of $k_{3}$. A mass 
(a)
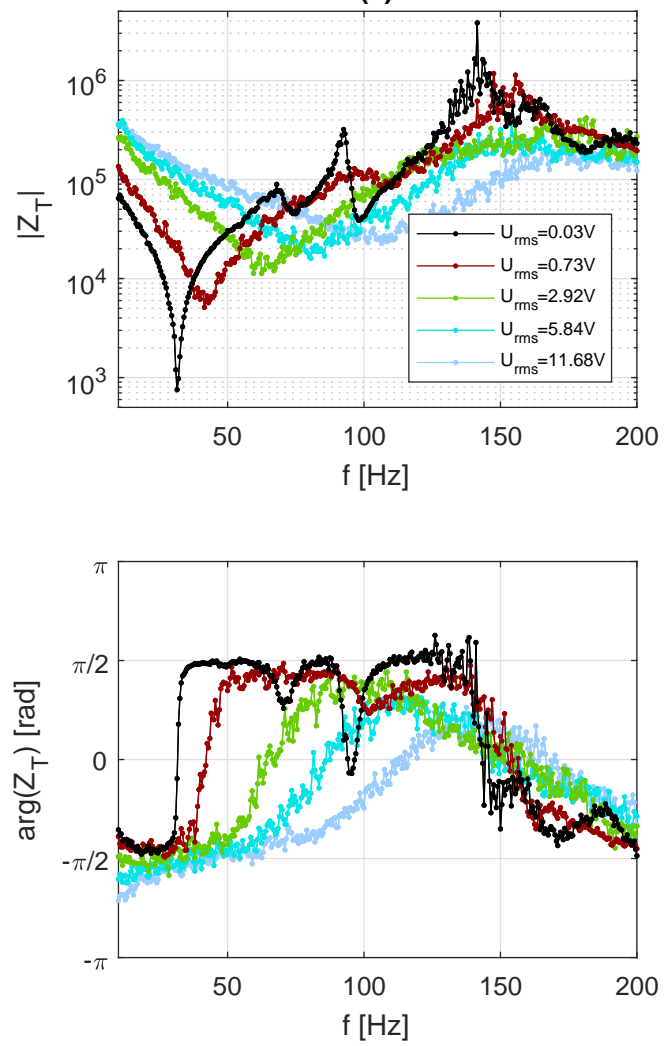

(b)
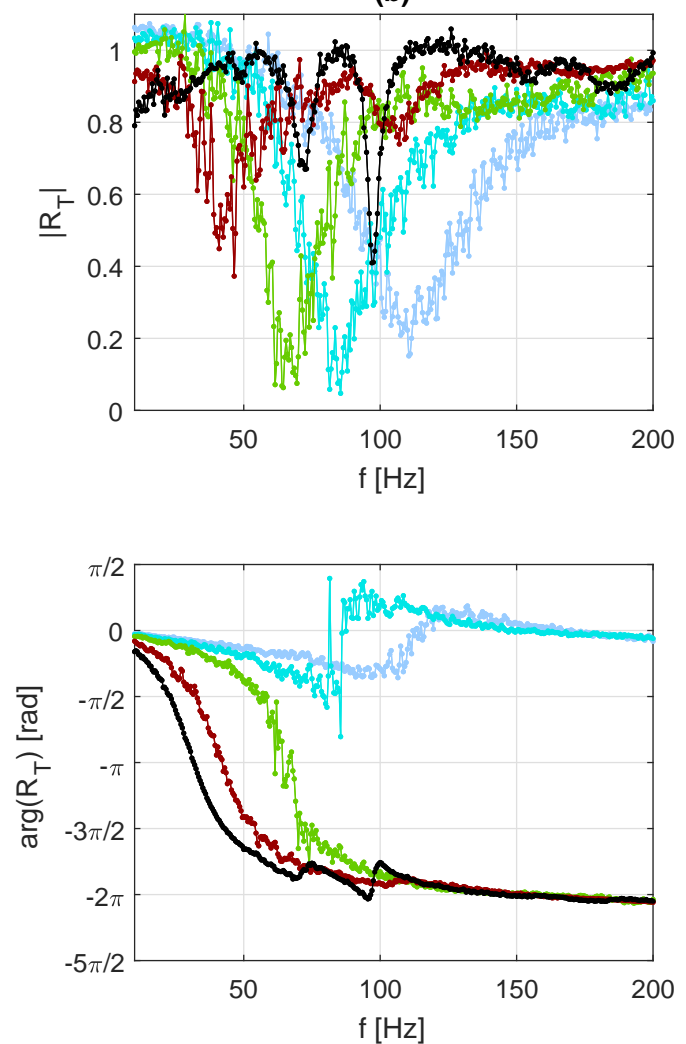

Figure 5: Identified acoustic characteristics of the NES for the excitation levels used in Fig. 3 (b): (a) Impedance $\left(Z_{\mathrm{T}}\right)$ and (b) associated reflection coefficient $\left(R_{\mathrm{T}}\right)$. Modulus (top) and phase (bottom).

term $m_{\mathrm{a}}$ has also been added to account for the mass of the air column resulting from the waveguide effect of the sliding system. Here $f_{0 \text { me }}$ represents the resonance frequency of the membrane alone (without pre-stress) and $f_{1 \mathrm{me}}$ is related to the pre-stress applied to the membrane. When no pre-stress is considered, $f_{1 \mathrm{me}}=f_{0 \mathrm{me}}$.

For a given excitation level, the acoustic impedance of the NES (close to the membrane) may be defined as :

$$
Z_{\mathrm{me}}=\frac{\hat{S}_{p_{\mathrm{me}} q_{\mathrm{me}}}}{\hat{S}_{q_{\mathrm{me}} q_{\mathrm{me}}}}
$$

where $\hat{S}_{q \mathrm{me} q \mathrm{me}}$ (respectively $\hat{S}_{p_{\mathrm{me}} q_{\mathrm{me}}}$ ) denotes the power spectral density (respectively cross spectral density) function of the volume velocity $q_{\text {me }}$ (respectively between the acoustic pressure $p_{\text {me }}$ and the volume velocity $\left.q_{\mathrm{me}}\right)$. The volume velocity $q_{\mathrm{me}}$ is obtained from the velocity of the membrane $\dot{u}_{\text {me }}$ as $q_{\text {me }}=\frac{S_{\text {me }}}{2} \dot{u}_{\text {me }}$. This definition is in accordance with the impedance quantity considered in Section 2.1.

The NES behavior being nonlinear, comparing model results to measured data requires to use temporal investigation of the nonlinear model. Provided that we use a time trajectory of the acoustic pressure $p_{\text {me }}$ which respects similar characteristics as in ex- 
perimental configuration, Eq. 10 can be numerically solved. The resulting trajectories are sufficient to estimate the NES impedance Eq. (13) via classical spectral estimation method.

As a gaussian white noise was fed to the source during the measurements, the modulus of the measured transfer function $H_{\mathrm{m}}$ (given Fig. 3) is sufficient to characterize the frequency content of the acoustic pressure in the vicinity of the membrane. $H_{\mathrm{m}}$ being defined at the microphone position, Eq. 5 is used to estimated the transfer function, $H_{\mathrm{m}}^{\mathrm{n}}$, between the control voltage $U$ and the acoustic pressure at the position $x_{\mathrm{n}}=x_{\mathrm{m}}+0.02$ ( $x_{m}$ has been corrected of the thickness of the sliding system) corresponding to $p_{\text {me }}$.

We then used the procedure described in 18 to generate time trajectories with a power spectral density function defined by $\left|H_{\mathrm{m}}^{\mathrm{n}}\right|^{2}$ on the frequency range $[10,200] \mathrm{Hz}$ and zero outside. These trajectories, corrected by the corresponding excitation level $U_{\mathrm{RMS}}$, were used as time trajectories of $p_{\mathrm{me}}$. Eq. (10) was then solved numerically using the Newmark method.

The following values of the parameters were chosen corresponding to the experimental set up: $\rho_{\mathrm{me}}=980 \mathrm{~kg} \cdot \mathrm{m}^{-3}, \nu=0.49, E=12.10^{5} \mathrm{~Pa}, h_{\mathrm{me}}=1.8 .10^{-4} \mathrm{~m}, R_{\mathrm{me}}=0.03 \mathrm{~m}$ and $m_{\mathrm{a}}=2.9710^{-4} \mathrm{~kg}$. Two parameters $f_{1 \mathrm{me}}\left(\geq f_{0_{\mathrm{me}}}\right)$ and $\eta(>0)$ were adjusted as $f_{1 \mathrm{me}}=40 \mathrm{~Hz}$ and $\eta=8.10^{-4}$ - corresponding to moderate pre-tension (as $f_{0 \mathrm{me}}=3.8 \mathrm{~Hz}$ ) and light damping.

The resulting impedance functions $Z_{\text {me }}$ brought back to the microphone position are plotted in Fig. 6 and Fig. 7 for the five excitation levels and compared to the corresponding ones identified experimentally by the proposed method. Also reported are the associated reflection coefficients. The viscoelastic model leads to a very good approximation of the measured data, except at the lowest amplitude $(0.03 \mathrm{~V})$ for which the impedance curve in Fig. 6 presents an anti-resonance peak close to $150 \mathrm{~Hz}$ which does not fit well with the experimental data. This might come from a coupling between the NES and the source setup, which would apparently shift the anti-resonance peak towards low frequencies. For all other excitation levels, the viscoelastic model seems quite adequate to reproduce the acoustic behavior of the NES. It describes correctly the shift of the resonance frequencies (hardening) and the spectral broadening which occurs around the resonance frequencies when the excitation level increases as estimated through the impedances and associated reflection coefficients.

\subsection{The NES as a family of Helmholtz resonators}

The impedances plotted in Fig. 6 have a shape suggesting that the system behaves like a resonant one degree of freedom system in the frequency range studied, however with level dependent parameters. An attempt is thus made to identify these parameter values expressed in quantities that permit comparisons with Helmholtz resonators, which are often used for sound control in this frequency range. At each excitation level, the behavior of the NES is then approximated by the impedance $Z_{\mathrm{H}}$ of a linear Helmholtz resonator :

$$
Z_{\mathrm{H}}=\mathrm{j} \omega M_{\mathrm{a}}+\frac{1}{\mathrm{j} \omega C_{\mathrm{a}}}+R_{\mathrm{a}}
$$

which is transformed at the microphone position as (Eq. (4)) :

$$
Z_{\mathrm{H}}^{\mathrm{th}}=\frac{Z_{\mathrm{H}}+\mathrm{j} Z_{c} \tan \left(k x_{\mathrm{m}}\right)}{\mathrm{j}\left(Z_{\mathrm{H}} / Z_{c}\right) \tan \left(k x_{\mathrm{m}}\right)+1}
$$



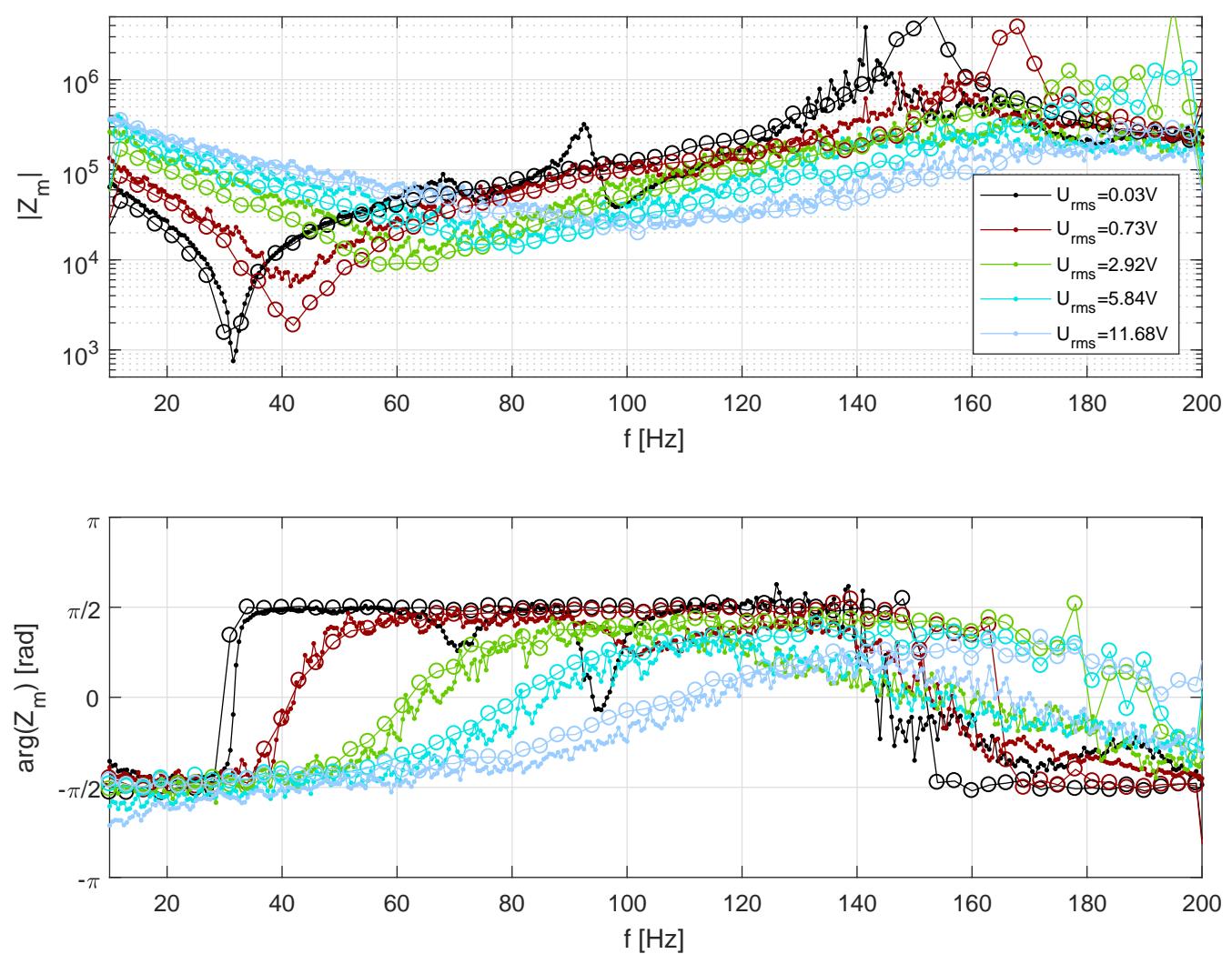

Figure 6: Viscoelastic model : Impedance $\left(Z_{\mathrm{T}}\right)$ identified from the measurement method (thick lines) and resulting from the viscoelastic model (lines and circle markers) for the excitation levels used in Fig. 3(b). Modulus (top) and phase (bottom).

where $M_{\mathrm{a}}$ is the acoustic mass, $C_{\mathrm{a}}$ is the acoustic compliance, $R_{\mathrm{a}}$ is the acoustic resistance. These parameters are then estimated from the measured data by solving the following minimization problem :

$$
\min _{\left(M_{\mathrm{a}}, C_{\mathrm{a}}, R_{\mathrm{a}}\right) \in \mathbb{R}_{+}^{3}} \sum_{l=1}^{F}\left|R_{\mathrm{H}}^{\mathrm{th}}\left(\omega_{l}\right)-R_{\mathrm{T}}\left(\omega_{l}\right)\right|^{2}
$$

where $R_{\mathrm{H}}^{\text {th }}$ is the reflection coefficient deduced from the Helmholtz model Eq. $15, R_{\mathrm{T}}$ is the estimated reflection coefficient (Eq. 3) and $\omega_{l}=2 \pi f_{l}$, with $f_{l}$ the frequency of the experiment number $l$.

This minimization problem has been solved for each excitation level using Matlab@ unconstrained nonlinear optimization solver "fminsearch". The results are reported in Table 1 The efficiency of the optimization process can be observed on Figure 8, where are compared $R_{\mathrm{H}}^{\text {th }}$ and $R_{\mathrm{T}}$ and on Figure 9 , where are compared the corresponding $Z_{\mathrm{H}}^{\text {th }}$ and $Z_{\mathrm{T}}$.

The parametric Helmholtz model leads to a very good approximation of the measured 

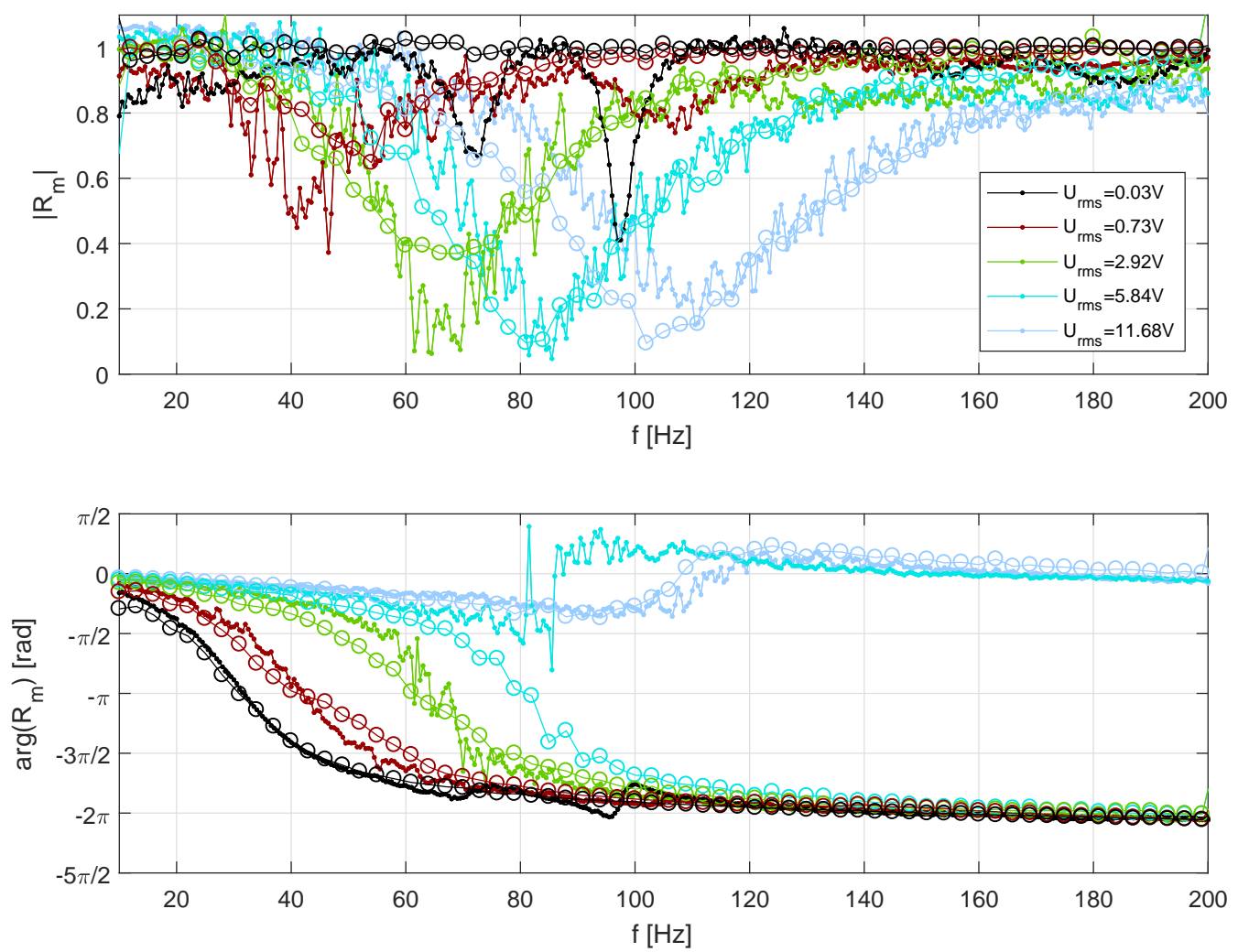

Figure 7: Viscoelastic model : Reflection coefficient $\left(R_{\mathrm{T}}\right)$ identified from the measurement method (thick lines) and resulting from the viscoelastic model (lines and circle markers) for the excitation levels used in Fig. 3.b). Modulus (top) and phase (bottom).

data, except at the lowest amplitude as mentioned earlier.

Looking to Table 1, the acoustic mass $M_{\mathrm{a}}$ is not much affected by the change of excitation level (values between 114 and $135 \mathrm{~kg} \mathrm{~m}^{-}$), compared to the large variations exhibited by the compliance $C_{\mathrm{a}}(180 \%)$ and the resistance $R_{\mathrm{a}}(55 \%)$. This is coherent for linear resonators, because the acoustic mass $M_{\mathrm{a}}$ of the Helmholtz model can be related to a physical mass $m_{\text {me }}$ described by the viscoelastic model by the relationship $M_{\mathrm{a}}=4 m_{\mathrm{me}} / S_{m}^{2}$. The parameters used for the simulations in section 3.3 correspond to $M_{\mathrm{a}}=133 . \mathrm{kg} \mathrm{m}^{-4}$, which is close to its values in Table 1 .

The compliance $C_{\mathrm{a}}$ and the resistance $R_{\mathrm{a}}$ are however much more level dependent.

\begin{tabular}{lcccccccccc}
\hline$U_{\mathrm{RMS}}[\mathrm{V}]$ & 0.03 & 0.70 & 1.5 & 2.9 & 4.4 & 5.8 & 7.3 & 8.8 & 10.2 & 11.7 \\
\hline$M_{\mathrm{a}}$ & 135 & 127 & 128 & 128 & 129 & 126 & 123 & 118 & 116 & 114 \\
$C_{\mathrm{a}}\left[\times 10^{-7}\right]$ & 1.83 & 1.11 & 0.74 & 0.46 & 0.39 & 0.28 & 0.24 & 0.22 & 0.20 & 0.18 \\
$R_{\mathrm{a}}\left[\times 10^{4}\right]$ & 0.16 & 0.57 & 0.93 & 1.42 & 1.82 & 2.23 & 2.49 & 2.70 & 2.89 & 2.96 \\
\hline
\end{tabular}

Table 1: Estimated Helmholtz resonator parameters: $M_{\mathrm{a}}, C_{\mathrm{a}}, R_{\mathrm{a}}$ as a function of $U_{\mathrm{RMS}}$. 

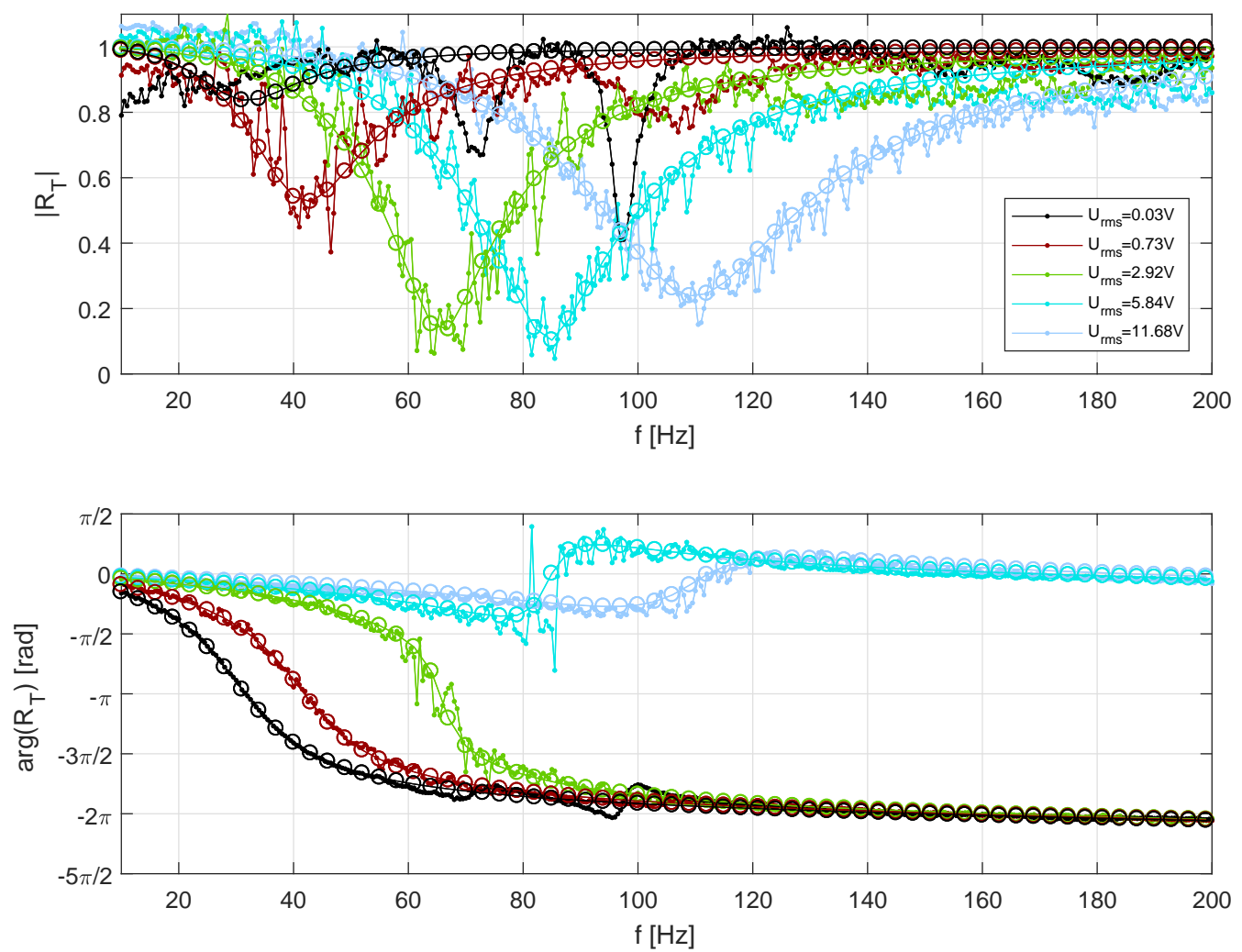

Figure 8: Helmholtz model: Reflection coefficient $\left(R_{\mathrm{T}}\right)$ identified from the measurement method (thick lines) and resulting from the Helmholtz model (lines and circle markers) for the excitation levels used in Fig. 3(b) . Modulus (top) and phase (bottom).

Their variations with respect to $U_{\mathrm{RMS}}$ can be analyzed considering the evolution of the resonance frequency and the quality factor defined as, respectively :

$$
f_{H}=\frac{1}{2 \pi \sqrt{M_{a} \times C_{a}}} \text { and } Q_{H}=\frac{\sqrt{M_{a} / C_{a}}}{R_{a}} .
$$

Variations of $f_{H}$ are plotted in Figure 10(a) and illustrate the hardening behavior of the NES mentioned previously. This hardening is not linear as it would be for an uncoupled purely cubic resonator excited at its resonance frequency. Figure 10(b) shows that the apparent quality factor is rather small, around 3 and is relatively constant after a sharp decrease at low excitation levels, until about $3 \mathrm{~V}$. The NES quality factors estimated here are realistic for actual Helmholtz resonators [19, although NES and Helmholtz resonators differ in their action. It means that Helmholtz resonators could be built with such quality factors. A small quality factor indicates a high dissipation, interpreted here as large extraction of energy from the system by several mechanisms: thermal dissipation in the viscoelastic membrane, acoustic radiation, and conversion to other frequencies than the excitation frequency [5].

Energy extraction can be seen more clearly on Fig, 10.(c) where the ratio $\mathcal{E}$ of extracted 

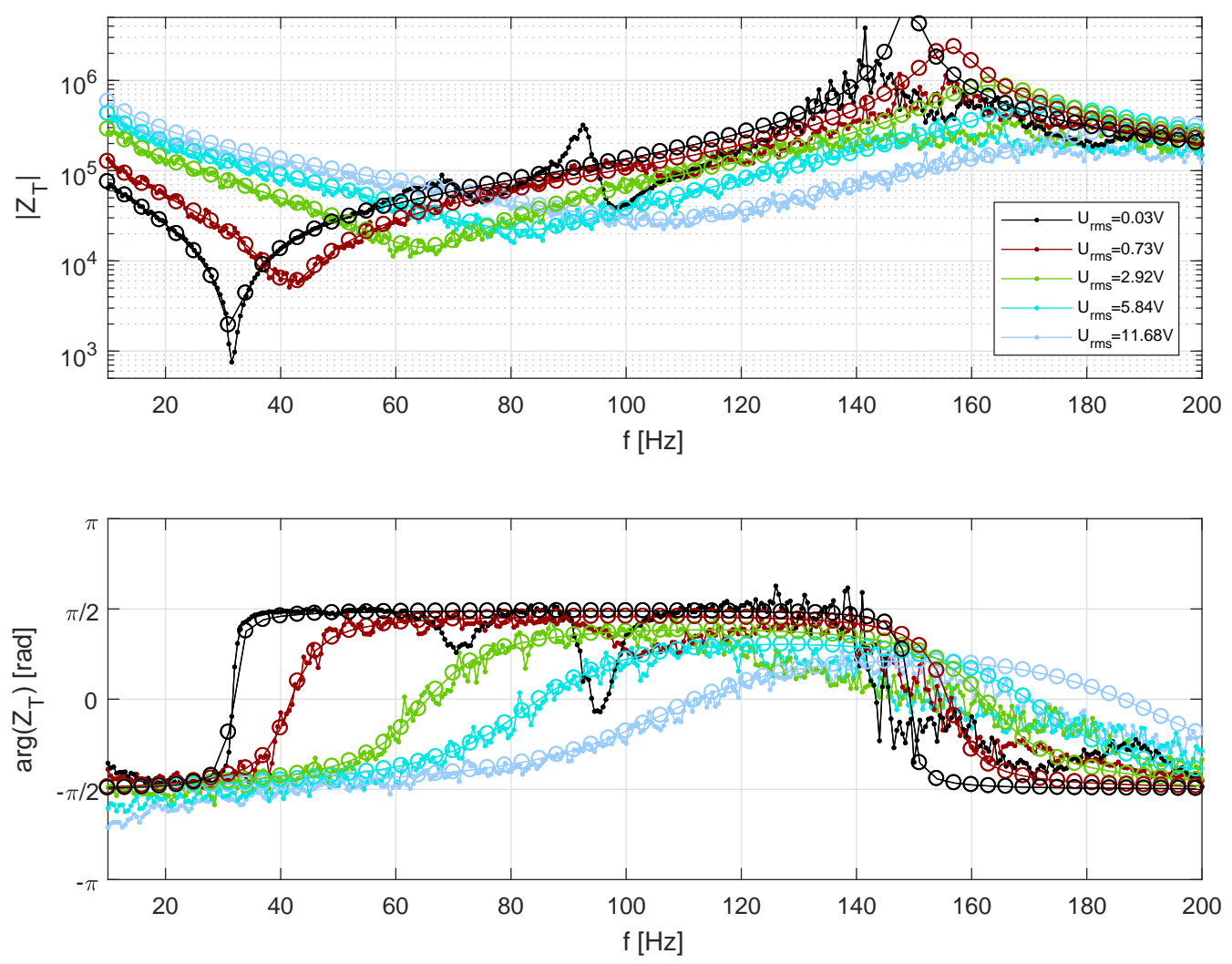

Figure 9: Helmholtz model: Impedance $\left(Z_{\mathrm{T}}\right)$ identified from the measurement method (thick lines) and resulting from the Helmholtz model (lines and circle markers) for the excitation levels used in Fig. 3. b) . Modulus (top) and phase (bottom).

energy over the frequency range of the source is defined by :

$$
\mathcal{E}=\left(f_{F}-f_{1}\right)^{-1} \int_{f_{1}}^{f_{F}}\left(1-\left|R_{\mathrm{T}}(2 \pi f)\right|^{2}\right) d f .
$$

is plotted, with $f_{1}=10 \mathrm{~Hz}$ and $f_{F}=200 \mathrm{~Hz}$.

At low levels the energy extraction $\mathcal{E}$ is quite small because the NES behaves like a linear Helmholtz resonator: it is efficient only close to its resonant frequency, with low damping. At higher levels $\mathcal{E}$ however reaches 0.35 , meaning that more than one third of the total incoming energy (spread over a wide frequency range) is extracted by the NES. It should however be noted that most of the energy extracted at these high levels corresponds to frequencies over $150 \mathrm{~Hz}$, part of which might be converted toward frequencies outside the analysis band. The shape and magnitude of the three curves Figure 10(c) are close. The Helmholtz model agrees best with the measurements in the upper half of the excitation level. The worse agreement between measurements and the viscoelastic model may come from the fact that it does not include the acoustic source in the model or that the effective NES stiffness doesn't fit well with the viscoelastic model. It illustrates the relevance of the Helmholtz model for the NES characterization. 
(a)

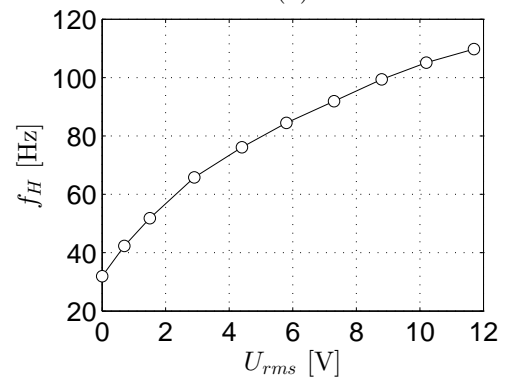

(b)

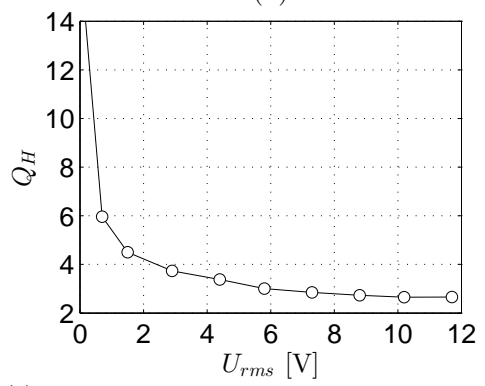

(c)

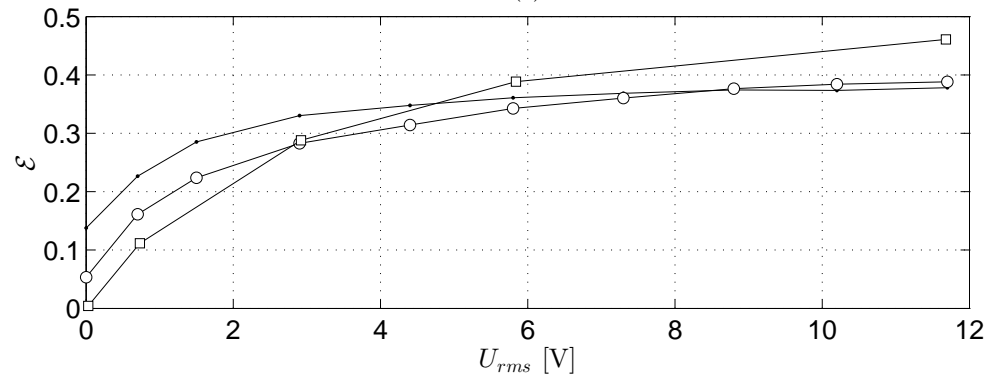

Figure 10: Helmholtz model fitting: Evolution of (a) the resonance frequency and (b) the quality factor (Eq. 17) versus excitation level. (c) Extracted energy $\mathcal{E}$ (Eq. 18 ) versus excitation level obtained from the measured reflection coefficient (dot markers), the viscoelastic model (square markers) and the Helmholtz model(circle markers).

This study shows that the NES may be described as a parametric Helmholtz resonator which parameters can be estimated with realistic values depending on the excitation level. It may prove useful for nonlinear absorber design, since the resonance frequency and attenuation as a function of sound level are key parameters 1 . In the framework of linearisation procedure [20, the amplitude level of the NES response could be also used as a parametric variable. This will be considered in future work.

\section{Conclusions}

The acoustic properties of a nonlinear vibroacoustic absorber have been investigated at low frequencies and high sound levels.

A measurement system based on impedance tube with a linear response up to $300 \mathrm{~Pa}$ in the $[10,200] \mathrm{Hz}$ frequency range has been built. It was tested and used with a bandlimited white source signal. In addition a multi-load technique has been developed to extract an apparent acoustic reflection coefficient. The method only requires a single microphone after a source characterization using a set of known loads.

The apparent reflection coefficient of the nonlinear vibroacoustic absorber was measured for increasing excitation levels. The results confirm a nonlinear behavior of the absorber. It is able to remove up to $90 \%$ of the incoming acoustic wave, around a frequency which is dependent on the source level. Unlike linear systems a fraction of the energy removed is probably converted into sound at frequencies above the excitation 
frequency band, in addition to the usual thermal dissipation mechanism. This frequency conversion has not been investigated yet, and is the focus of ongoing work.

A nonlinear viscoelastic model previously published was found adequate to describe the nonlinear characteristics of the NES measured here, based on a simple latex membrane. From the same acoustic measurements, a family of equivalent Helmholtz resonators with level-dependent parameters was identified. At each excitation level, the corresponding model captures most of the action of the nonlinear absorber with realistic parameter values: the identified mass is almost constant with the excitation level and close to the value used in the nonlinear viscoelastic model.

This study showed the good efficiency of the method and setup proposed for high level, low frequency measurements of non-linear loads such as the one considered here. It paves the way for future works about other NES devices.

\section{Acknowledgements}

This works has been done in the framework of the labex MEC, part of the Idex A*MIDEX. The authors acknowledge SATT Sud-EST (France) for its financial support.

\section{Appendix A}

In this appendix, the principle of the method used to estimate the source characteristics $H_{a e}$ and $Z_{\mathrm{s}}$ from $N(\geq 2)$ known loads is briefly summarized.

Following Desmons [15], a least square method is developed. The target of the minimization is the reflexion coefficient $R_{\mathrm{T}}$ because it is the quantity of interest for the characterization of the energy extracted by NES. For this purpose, the following cost function is used :

$$
J_{\mathrm{c}}=\sum_{n=1}^{N}\left(\frac{\left|R_{\mathrm{T}_{n}}^{\mathrm{th}}-R_{\mathrm{T}_{n}}\right|^{2}}{\left|R_{\mathrm{T}_{n}}^{\text {th }}\right|^{2}}\right)
$$

where $R_{\mathrm{T}_{n}}^{\text {th }}$ is the theoretical reflection coefficient deduced from the impedance model of the known load number $n$ and $R_{\mathrm{T}_{n}}$ is the reflection coefficient deduced from Eq. (3) with the measured transfer function $H_{\mathrm{m}_{n}}$. The dependency of $R_{\mathrm{T}_{n}}$ with respect to $H_{\mathrm{ae}}$ and $Z_{\mathrm{s}}$ appears from Eq. (3).

Separating the characteristics of the source into real and imaginary parts :

$$
H_{\mathrm{ae}}=H_{\mathrm{ae}}^{\mathrm{R}}+j H_{\mathrm{ae}}^{\mathrm{I}} \text { and } Z_{\mathrm{s}}=Z_{\mathrm{s}}^{\mathrm{R}}+j Z_{\mathrm{s}}^{\mathrm{I}},
$$

the optimum values of $H_{\mathrm{ae}}$ and $Z_{\mathrm{s}}$ are obtained solving the minimization problem expressed as :

$$
\min _{\left(H_{\mathrm{ae}}^{\mathrm{R}}, H_{\mathrm{ae}}^{\mathrm{I}}, Z_{\mathrm{S}}^{\mathrm{R}}, Z_{\mathrm{S}}^{\mathrm{I}}\right) \in \mathbb{R}^{4}} J_{\mathrm{c}} .
$$

This non-quadratic minimization problem is solved for each frequency with Matlab@ unconstrained nonlinear optimization solver "fminsearch" (based on the Nelder-Mead simplex algorithm). At the smaller frequency, the starting point of the algorithm is obtained from Eqs. (9). The frequencies are then considered in increasing order, with a starting point defined by the optimum obtained at the previous frequency. 


\section{Appendix B}

In this appendix, a brief description of the source design is given.

(a)

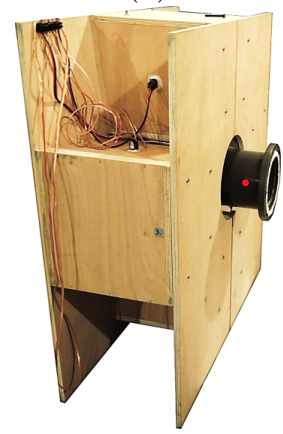

(b)

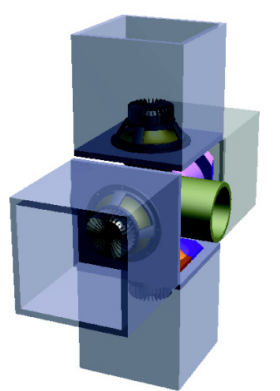

Figure 11: (a) Picture of the source. (b) Scheme showing the four loudspeakers

A particular source (Fig. 11) was designed for the low frequency range (typically 50 to $200 \mathrm{~Hz}$ ), in order to expose the DUT to high pressure levels. Attention was paid to the linearity and the smooth frequency response of the source. It is composed of four loudspeakers (type Beyma 10LW30/N, diam $0.25 \mathrm{~m}$ ) connected perpendicularly at the mid length of a tube with length $0.71 \mathrm{~m}$ and diameter $D_{t}=0.175 \mathrm{~m}$. The rear face of each loudspeaker is enclosed in a $37 \ell$ air-tight box. The front side of each loudspeaker is connected to the tube by smooth 3D-printed horns with initial diameter $0.25 \mathrm{~m}$ and final diameter $0.1 \mathrm{~m}$. The four loudspeakers are connected at the same abscissa (see Fig. 11(b)), and contribute equally to the volume velocity of the source. The two ends of the source tube can be fitted by a lid or by any load. For the present study, the left side of the source is closed by an air-tight PVC disc. Only the right part is changed and the microphone is localized $0.035 \mathrm{~m}$ away from the DUT (resulting in $x_{\mathrm{m}}=0.035 \mathrm{~m}$ ). The air-tightness of the source is critical and has been carefully checked.

\section{Appendix C}

In this appendix, the calibration process is presented. Seven known loads are used (given Table 2). They are made of PVC tubes having the same diameter as the source tube, with a first side end open (for the connection with the source) and the second side end depending on the load number. The second side end can be open, or closed, or terminated by a drilled lid. They are described by means of their theoretical impedances at $x=L+x_{m}$. Theoretical impedances are set to zero for a closed tube, and for an unflanged open tube, we adopt the classical approximation (21] Eq. (12.133)) recalled Table2 2 For the last load, number 7 , terminated by a drilled lid, we express the theoretical impedance at the microphone position as:

$$
Z_{7}=Z_{\mathrm{rad}}+Z_{\text {cor }}
$$


where $Z_{\text {rad }}$ is approximated by a flanged plane piston of radius $R_{7}$ (21] Eq. (12.126)), as:

$$
Z_{\mathrm{rad}}=Z_{\mathrm{c}}\left(\frac{1}{2}\left(k R_{7}\right)^{2}+j \frac{8}{3 \pi} k R_{7}\right)
$$

and $Z_{\text {cor }}$ is an impedance correction including two inertial terms as:

$$
Z_{\mathrm{cor}}=j k \frac{\rho c}{\pi R_{7}^{2}}\left[e_{7}+R_{7}\left(\frac{8}{3 \pi}-0.3525 \pi \alpha+0.0643 \pi \alpha^{2}\right)\right] .
$$

The first term corresponds to the acoustic mass of the fluid within the cylindrical aperture of the termination, of thickness $e_{7}$. The second term (21 Eq. (7.163)) corresponds to the discontinuity in cross section between the aperture and the tube, with $\alpha=R_{7} / R_{t}$.

The first six known loads, named reference loads, are employed to estimate $Z_{s}$ and $H_{a e}$ as described in Appendix A and one, number 7, is a test load.

\begin{tabular}{cclc}
\hline $\begin{array}{c}\text { Load } \\
\text { number }\end{array}$ & $\begin{array}{c}\text { Length } \\
L(\mathrm{~m})\end{array}$ & End & $\begin{array}{c}\text { Impedance } \\
\text { at } x=L+x_{m}\end{array}$ \\
\hline 1 & 0 & open & $Z_{1}=Z_{\mathrm{c}}\left(\frac{1}{4}\left(k \frac{D_{\mathrm{t}}}{2}\right)^{2}+j 0.6133 k \frac{D_{\mathrm{t}}}{2}\right)$ \\
2 & 0 & PVC plug & $Z_{2}=0$ \\
3 & 0.314 & wood plug & $Z_{3}=0$ \\
4 & 0.403 & PVC plug & $Z_{4}=0$ \\
5 & 0.8 & open & $Z_{5}=Z_{\mathrm{c}}\left(\frac{1}{4}\left(k \frac{D_{\mathrm{t}}}{2}\right)^{2}+j 0.6133 k \frac{D_{\mathrm{t}}}{2}\right)$ \\
6 & 0.8 & PVC plug & $Z_{6}=0$ \\
7 & 0.314 & $\begin{array}{l}\text { wood plug with centered } \\
\text { hole radius } R_{7}=0.03 \mathrm{~m}\end{array}$ \\
& & $Z_{7}$ \\
& & \\
\hline
\end{tabular}

Table 2: Characteristics of the known loads.

Using Eq. (4), the reference load impedance at the microphone location $x=0$ is obtained for the known loads. Using $H_{\mathrm{ae}}$ and $Z_{\mathrm{s}}$ fitted to the the six reference loads measurements, we measure the reflection coefficient $R_{\mathrm{T}}$ of the test load.

In our experiments, the worst error on $R_{\mathrm{T}}$ among all the test frequencies is below $5 \%$. We checked that any of the reference load relative error is below this value. We consider this worst error to be representative of the uncertainty of the measurements.

\section{References}

[1] R. Bellet, B. Cochelin, P. Herzog, P.-O. Mattei, Experimental study of targeted enrgy transfer from an acoustic system to a nonlinear membrane absorber, Journal of Sound and Vibration 329 (14) (2010) 2768-2791.

[2] R. Mariani, S. Bellizzi, B. Cochelin, P. Herzog, P.-O. Mattei, Toward an adjustable nonlinear low frequency acoustic absorber, Journal of Sound and Vibration 330 (22) (2011) 5245-5258.

[3] A. F. Vakakis, O. V. Gendelman, L. A. Bergman, D. M. McFarland, G. Kerschen, Y. S. Lee, Nonlinear targeted energy transfer in mechanical and structural systems, Vol. 156, Springer Science \& Business Media, 2008. 
[4] R. Bellet, B. Cochelin, R. Côte, P.-O. Mattei, Enhancing the dynamic range of the targeted energy transfer in acoustics using several nonlinear membrane absorbers, Journal of Sound and Vibration 331 (26) (2012) 5657-5668.

[5] R. Côte, M. Pachebat, S. Bellizzi, Experimental evidence of simultaneous multi-resonance noise reduction using an absorber with essential nonlinearity under two excitation frequencies, Journal of Sound and Vibration 333 (20) (2014) 5057-5076.

[6] J. Shao, B. Cochelin, Theoretical and numerical study of targeted energy transfer inside an acoustic cavity by a non-linear membrane absorber, International Journal of Non-Linear Mechanics 64 (2014) 85-92

[7] J.-P. Dalmont, Acoustic impedance measurement, part i: A review, Journal of Sound and Vibration 243 (3) (2001) 427-439.

[8] A. Seybert, D. Ross, Experimental determination of acoustic properties using a two-microphone random-excitation technique, The Journal of the Acoustical Society of America 61 (5) (1977) 13621370 .

[9] J. Chung, D. Blaser, Transfer function method of measuring in-duct acoustic properties. i. theory, The Journal of Acoustical Society of America 68(3) (1980) 907-913.

[10] J. Chung, D. Blaser, Transfer function method of measuring in-duct acoustic properties. ii. experiment, The Journal of Acoustical Society of America 68(3) (1980) 914-921.

[11] M. Abom, H. Bodén, Error analysis of the two-mircophone measurements in ducts with flow, The Journal of Acoustical Society of America 83 (6) (1988) 2429-2438.

[12] V. Gibiat, F. Laloë, Acosutic impedance measurements bu the two-microphone-three-calibration (tmct) method, Journal of Acoustical Society of America 88(6) (1990) 2533-2545.

[13] R. Boonen, P. Sas, W. Desmet, W. Lauriks, G. Vermeir, Calibration of the two microphone transfer function method with hard wall impedance measurements at different reference sections, Mechanical Systems and Signal Processing 23 (5) (2009) 1662-1671.

[14] M. Prasad, A four load method for evaluation of acoustic source impedance in a duct, Journal of Sound and Vibration 114 (2) (1987) 347-356.

[15] L. Desmons, J. Hardy, A least squares method for evaluation of characteristics of acoustical sources, Journal of Sound and Vibration 175 (3) (1994) 365-376.

[16] H. Bodèn, On multi-load methods for determination of the source data of acoustic one-port sources, Journal of Sound and Vibration 180 (5) (1995) 725-743.

[17] H. Bodèn, Error analysis for the two-load method used to measure the source characteristics of fluid machines, Journal of Sound and Vibration 126 (1) (1988) 173-177.

[18] F. Poirion, C. Soize, Simulation numérique de champs vectoriels stochastiques gaussiens homogènes et non homogènes, La Recherche Aérospatiale 1 (1989) 41-61, F. Poirion, C. Soize, Numerical simulation of homogenous and non homogenous, gaussian stochastic vectorial fields, Aerospace Research 1 (1989) 41-61.

[19] P. Herzog, A. Soto-Nicolás, F. Guéry, Passive and active control of the low-frequency modes in a small room, in: Audio Engineering Society Convention 98, Audio Engineering Society, 1995.

[20] L. Socha, Linearization methods for stochastic dynamic systems, Vol. 730 of Lecture Notes in Physics, Springer, Berlin Heidelberg, 2008

[21] A. Chaigne, J. Kergomard, Acoustics of Musical Instruments, Springer, 2016. 